\title{
Measuring Quality of Life in Urban Areas: Toward an Integrated Approach
}

\author{
Jamal Al-Qawasmi* \\ Department of Architecture, King Fahd University of Petroleum \& Minerals (KFUPM), Saudi Arabia
}

Submission: March 16, 2020; Published: July 08, 2020

*Corresponding author: Jamal Al-Qawasmi, King Fahd University of Petroleum \& Minerals (KFUPM), Dahran, Saudi Arabia

\section{Abstract}

The paper examines the various aspects involved in contextualizing and measuring quality of life (QOL) in urban areas. The paper reports on an on-going research project that aims to establish multi-dimensional assessment system to measure QOL in Saudi Arabian cities. To develop a localized QOL measure that capture local aspects of QOL without losing the potential to compare data between different cities or regions, the paper critically analyzed and examined a wide range of conceptual and methodological issues related to QOL assessment and measurement The study pointed out the need to adopt a multi-dimensional integrated approach that uses both a top-down and a bottom-up approach and incorporate secondary/objective data as well as primary data obtained through self-report surveys. An integrated framework to contextualize, operationalize and measure QOL in the Saudi context has been developed based on critical analysis of literature.

Keywords: Quality of life; Quality of urban life; Urban areas; Cities; Saudi Arabia

\section{Introduction}

Quality of Life (QOL) is defined as the general well-being of people and the quality of the environment in which they live. Since the 1940s, quality of life (QOL) has been recognized as an important concept in several academic disciplines such as urban studies, sociology, economics, environmental sciences, psychology, political science, marketing, among others. QOL studies vary according to their scope, purpose, field, and approach. Different disciplines are interested in various aspects of quality of life, and thus the QOL construct has been conceived and measured differently.

Studies on QOL in cities emerged as a tool to track changes in different aspects of life and assess types of development that fit people needs and priorities. QOL studies in cities have been driven by municipal programs, community programs, business community programs and transnational city networks programs. This wide scope makes QOL research a powerful, practical, and effective tool to test and measure the impact of urban and socioeconomic development policies and actions.

Saudi Arabia had invested heavily in developing its urban centers and improving quality of life for citizens. However, there is lag in developing metrics to measure QOL in its cities and evaluating the efficiency of the deployed development policies and their impact on people. A research project titled "Developing a tool to Assess and Monitor Urban Quality of Life in Saudi Arabia
Cities" has started late early 2019 aiming at setting up criteria suitable for measuring QOL in Saudi Arabian cities. In particular, the project aims to identify and establish appropriate QOL domains and indicators and the framework that related and link them together. This paper reports on the first phase of the project in which a comprehensive analysis and review of QOL literature was conducted. The paper examined the evolution of QOL concept and relevant research approaches to establish a framework to develop a local QOL assessment system.

\section{Defining and Contextualizing QOL}

\section{Emergence and evolution of QOL concept}

Although QOL is a contemporary construct, the idea of having a good life has been discussed by various thinkers throughout history and across cultures including Plato, Aristotle, Kant, and in the Islamic civilization as early as Alfarabi with his concept of the virtuous city (c.940). The term "quality of life" emerged in USA first after the WWII. It was used to describe the effect of material affluence on people's lives and to emphasize basic human needs and the reduction of poverty. Research on QOL moved significantly forward as of the 1960s by the Social Indicators Movement [1,2]. Environmental indicators were received more attention later in the 1960s when environmental issues and their impact on human health became obvious, and an issue of public debate [3]. Through time, the QOL concept was expanded to encompass education, 
health and welfare, social security, working conditions, and economic growth. Currently, QOL has been a globally accepted aspiration for individuals, communities, and nations [4,5].

Researchers and scientists from various fields, in addition to governmental agencies showed great interest in the QOL topic. Overtime it became an interdisciplinary field of study. Healthcare professionals, political researchers, policy makers, planners, economist, environmentalist, and sociologists have come up with different definitions and approaches to explore how to measure and improve quality of life in different societies and environments. However, reaching conclusions or building decisions based on these interdisciplinarity studies remain difficult due to difference in perspectives and methods adopted. Therefore, a recent trend is to develop a unified QOL assessment system that incorporates economic, social, and environmental indicators which are known nowadays as QOL indicators [3].

To measure quality of life, one must have a theory of what makes up a good life. However, literature shows that although it has been a widely used concept, QOL is has more than 100 definitions and even more interpretations of QOL data depending on the context and scope of the research and its aim or purpose. QOL is still considered to be vague and elusive, and difficult to define or measure [6]. For some, QOL is concerned with personal well-being and satisfaction or happiness, while for others, it is concerned with living conditions of a place. As a result, there is a wide range of theories of what constitutes a "good life" or a "good urban setting".

It is well established that there is no universally accepted definition of QOL [6-8]. However, the variety of definitions and meanings of QOL is not a sign of conceptual weaknesses, but a sign of the complexity of the concept and interdisciplinarity of the field. It is generally accepted that the nature and interpretations of the QOL concept reflect the specific scientific goals of the research project, the theoretical models or academic orientations adopted, and the place and context of the study. Even for the same or similar theories and concepts, different operationalization may be adopted with each revealing different attributes and dimensions of QOL. This situation highlights the need to contextualize the QOL concept in our project [7]

Examining the large set of definitions provided in QOL literature, one can clearly notice that these definitions of QOL represents one of two tendencies: those refer to and focus on the objective environment and those refer to and focus on how the individuals perceive, feeling, experience life objective conditions. These two tendencies resulting from the two different lines of measurement strategies - one focusing on individual subjective well-being, while the other on objective urban quality of life [7]

Based on an extensive review of literature on QOL, Mulligan et al. [9] broadly defined QOL as the satisfaction that a person receives from surrounding human and physical conditions [9]. This definition seems appropriate to start with in measuring QOL in urban areas as it recognizes the impact of physical conditions of the place.

\section{Confusions with related concepts}

Literature shows that the QOL concept is conflated with several other concepts, and in many cases the different concepts are used interchangeably although there are differences or at least nuance among them. These related concepts include life satisfaction, happiness, well-being, standard of living, health status and living conditions $[6,8]$. This practice highlights the lack of consensus on QOL definition. When dealing with QOL, one should distinguish between the terms and use them accurately.

Standard of living refers to how much and how well a society can provide goods and services to its citizens. The common measures contain gross domestic product (GDP) per capita, birth rates, life expectancy, mortality rates, fertility rates, literacy level, living density, population per dwelling and the so. QOL on the other hand, is the product of the interplay among different conditions including social, economic, health, and environmental ones which affect human and social development. Thus, QOL covers wider domains and more dimensions than that of living standards.

A similar approach to the study of quality of life depends on the "subjective well-being" concept. It is defined as a subjective state of a person obtained from his/her evaluation of life and expressed as happiness or satisfaction. Apparently, the "subjective well-being" concept captures a big trunk of QOL and is widely used in QOL studies. The United Nations even treats them equivalent because its QOL indexes include many criteria which are used to determine well-being [7].

The concept of life satisfaction has been always mixed with QOL too. Several scholars in 1970s and 1980s defined "life satisfaction" as the quality of life experience [8]. Felce \& Perry [8] pointed out that QOL "is the sum of a range of objectively measurable life conditions experienced by an individual" distinguished it from satisfaction which is considered as "subjective responses to such conditions" (p. 54). Other studies defined satisfaction as individuals' adaptation toward specific life conditions [10]. Therefore, some scholars consider life satisfaction and QOL the same [10], while others regard life satisfaction as part of QOL, depending on their definition of QOL. However, it is important when carrying up any QOL to distinguish between the two concepts and define what the study concerns about.

The project has also reviewed literature related to welfare and happiness concepts and how they differ from and overlap with the QOL concept $[11,12]$. The use of different concepts interchangeably with QOL shows lack of consensus on defining QOL and highlight the interdisciplinarity and multidimensionality nature of QOL.

\section{Research Approaches to QOL}

QOL research shows variety of research approaches some of 
which are dedicated to the study of QOL, while others are linked implicitly to QOL research. Examples of the latter includes the approaches used in the areas of social indicators movement, urban amenity, and livability studies. Joseph Sirgy [13] has reviewed over 13 theories that were used to integrate and unify ideas in QOL research. In this regard, four paradigms of QOL research have been identified to be influential.

\section{Paradigm 1: Utilitarianism}

The utilitarian theory holds that QOL means nothing but the desire and satisfaction of individuals. A society can be called a good society only if it provides the maximum satisfaction or positive experiences for its citizens. The most common human desire is economic success; therefore, utilitarianism mainly focuses on the materialistic achievement. Typical utilitarian definitions of QOL are as follow:

"feeling of well-being, fulfillment or satisfaction resulting from factors in the external environments" [14].

"individuals' perceptions of their position in life in the context of the culture and value systems in which they live and in relation to their goals, expectations, standards and concern" [15].

\section{Paradigm 2: Human development paradigm}

Initiated by the United Nations Development Program (UNDP), the human development theory developed a wider perspective which incorporates ideas from ecological economics, sustainable development, welfare economics, and feminist economists. Instead of measuring well-being and detecting economic growth only, they optimize well-being by deploying social capital to optimize values of human capital in an economy. According to this perspective, the value of society doesn't lie in the attainment of personal happiness but lies in its capability to help its citizens to develop virtues, become artistic, and attain wisdom. The importance of freedom is emphasized as a key element in the definition of the good society. A society is judged by whether policies, procedures and structures can promote moral responsibility, rational choice, and other features of full human development. In this paradigm, special attention is paid to institutions such as markets, politics, public rituals, family, school, and work. A significant contribution of this paradigm is that it puts sustainable development (environmental, social, and economic sustainability) as a key part of a good society. The following are some typical definitions:

"the product of the interplay among social, health, economic and environmental conditions which affect human and social development" [16].

"the general well-being of people and the quality of the environment in which they live" [15].

\section{Paradigm 3: Capability paradigm}

The capabilities paradigm, firstly developed by the Nobel laureate in economics A. Sen, is related to human development theory [5,17]. Since living is seen as a combination of various doings and beings, quality of life, from this perspective, is conceived as the individual capabilities to achieve valuable functioning's which refers to what people can choose to do or to be. For example, a person is in good health; a person has self-respect; a person is socially integrated in a community. Recognizing individual's ability to pursue his/her goals is an important assumption in this paradigm. Developing virtues and loyalties, becoming skilled and artistic, and attaining wisdom are also indispensable for human beings. If people in a society have high capability to reach these aims, they may be regarded to have high QOL. The list and nature of important capabilities vary across studies.

\section{Paradigm 4: Opportunity paradigm}

The QOL in the opportunity paradigm is broken down into four parts, namely an outer-quality-of-life-chances (the livability of the environment for a person), an inner-quality-of-life-chances (a person's life-abilities), an outer-quality-of-life-results (the utility of a person's life), and an inner-quality-of-life-results (a person's experience of his life as a good one) [18]. Oriented this way, what government needs to do to improve QOL is to develop policies which can create opportunities for human needs to be met. This includes creating conditions to increase the likelihood that people will effectively take advantage of these opportunities. Obviously, the social dimension (relational and societal characteristics) is added to this theoretical framework. Social cohesion is seen as a crucial feature of good society. Concepts like equality, equity, freedom, and solidarity are deeply discussed to examine how they influence QOL directly or indirectly. From this perspective, all we need "to create an environment and chances for people to improve QOL" is to build a sustainable environment. A typical definition of QOL will be:

"The quality of the living environment or the availability of good educational and recreational facilities of a high sense of security and safety".

\section{Urban QOL: The Role of Place}

While there is consensus about the intrinsic meaning of QOL, literature points out that it is the spectrum of living conditions of urban areas and particularities of these areas that generates greater divergences. Exploring urban QOL necessitates examining the relationship between the material and immaterial aspects of life and their contribution to QOL. This section addresses QOL as it relates to places and urban settings.

In an urban setting, people simultaneously experience and interact with several environmental factors such as natural, built, social and economic. As such, assessing QOL in urban settings should cover the environmental, social, and economic aspects of the urban environment. QOL research has extensively examined the social and economic aspects of life and their impact on QOL. However, we know very little about how the physical environment, and its interaction with socio-economic factors, impact QOL in urban areas. 
In his study of QOL in cities, Rogerson (1999) classified QOL studies into three typologies [19]. In Type A studies, QOL is investigated with emphasis placed on places and their characteristics (environmental, social, and economic). The focus in Type C studies is on the personal aspects of QOL; that is QOL of a place is investigated as subjective phenomenon that reflects the perceptions and life experiences of the residents of the place. Type B studies, on the other hand, combine aspects of both Type $\mathrm{A}$ and $\mathrm{C}$. The focus in this type of studies is placed on places and their objective characteristics, while priorities and weights of these objective characteristics are derived through self-report surveys, and thus some personal aspects of QOL is incorporated [19]. Here it worth mentioning that type B of QOL studies overlap with the well-known concept of "sense of place". The Dictionary of Human Geography (2009) defines the sense of place as "the attitudes and feelings that individuals and groups hold vis-a`-vis the geographical areas in which they live. It further commonly suggests intimate, personal and emotional relationships between self and place" [20] [p. 676].

To understand the complex urban QOL concept, it is necessary to examine it through research of Type B; that is to explore the mechanisms through which objective characteristics of place and subjective evaluations are interacting in a specific context/ setting. As discussed in section 5 below, literature shows that the exclusive use of objective indicators for the study of citizens' satisfaction with their city was a limited approach. At the same time, overemphasize subjective aspects may limit the ability to compare data between different cities or regions. Thus, there is a need to adopt both approaches (objective and subjective) to measure/assess QOL [21,22]. Based on literature we developed a model, depicted in figure, that integrates the various aspect of urban environment to contextualize the QOL concept.

\section{Operationalizing and Measuring QOL}

\section{Conceptualizing and operationalizing QOL}

While there is lack of consensus about the definition of QOL, however, one can identify a general procedure to follow in contextualizing QOL and developing an assessment system to measure it, as depicted in figure 1. The first step in defining and contextualizing QOL is to develop a framework that conceptualizes and describes the core dimensions that constitute QOL. The next step is to operationalize those aspects for accurate measurement. Very often, the process starts with a long list of core dimensions (attributes) which are subdivided into several sub-categories. The core dimensions are called domains which are the main attributes/aspects of life and usually contain several elements/ sub-categories which are called indicators [23]. For instance, the well-known WHOQOL instruments breaks QOL into "physical", "psychological", "social relationships" and "environment" domains. These domains are subdivided into subcategories or indicators that enable researchers to measure and quantify domains. In many cases, an indicator is sub-divided into a set of sub-indictors that specify the variables or parameters that will be measured. Both domains and indicators are joined by a theoretical framework, known as the conceptual map, that describes the relationship between the various domains, indicators, and sub-indicators $[7,23]$. This framework is a fundamental part of contextualizing the QOL concept for use in specific region. As such, it needs special attention and should be done carefully as it defines and conceptualizes what are the proper aspects that will be used as domains, indicators and sub-indicators, as well as the degree and shape of linkages between these domains and indicators, based on the purpose of the QOL assessment system and the particularities and needs of the region.

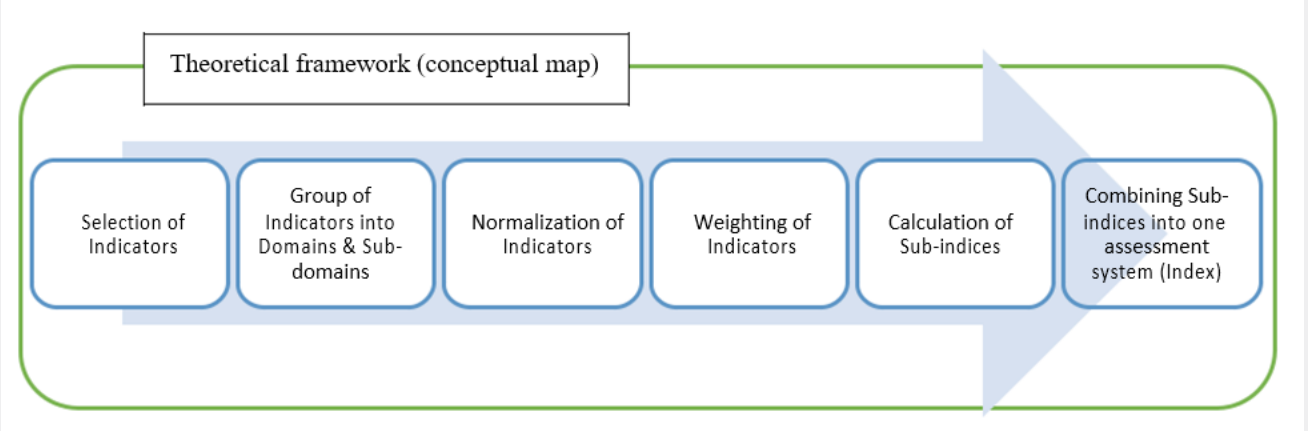

Figure 1: A general framework to contextualize QOL and develop an assessment system to measure it (source: author).

\section{Selecting domains and indicators}

\section{QOL domains}

QOL domains vary in number and terminology due to differences among research studies, although they may represent the same concepts in most of cases. To contextualize QOL, a set of guidelines should be adopted in constructing QOL domains. Among the different dimensions/elements nominated to be used as domains for a QOL assessment system, only those achieving certain criteria are to be accepted as successful domains. Domains of QOL must encompass the totality of life experience not just some component parts. On the other hand, each domain must 
encompass a substantial but discrete portion of the QOL construct. QOL domains must be potentially neutral, widely acknowledged by majority of any population, and have positive contribution to QOL construct. In addition, a good QOL domain should allow us to measure it using both subjective and objective methods. For the domains which seem subjective, they should have both cognitive and affective components, which help avoid biases [10] (Figure 1).

\section{QOL indicators}

To conceptualize and operationalize QOL domains, there is a need to break them down to measurable units/variables which usually are called indictors. QOL indicators are constructs that composed of measurable variables that quantify and communicate important QOL issues and trends.

QOL indicators usually measure aspects that are agreed to be important aspects of QOL in a specific region. Ideally, an indicator should incorporate all attributes which it measures although it is a very difficult job. An indicator that is meaningful and useful usually reflects a combination of idealism (what is to be measured) and pragmatism (what can be measured). This makes the selection of suitable QOL indicators a difficult task. Selection of indicators is usually based on the research scope and emphases; therefore, they are always weighted and ranked differently.

Selecting QOL indicators for specific context should meet certain criteria, which may differ based on the goal and scope of the research. However, research points out that regardless of the aim of the research study, there are some fundamental principles that should be considered when selecting QOL indicators, such as: Purity (filtering out extraneous factors, policy relevant); validity (relevant to quality of life); availably (available on a regular basis,); predictability (can predict the direction of trends); simplicity and understandability (easy to interpret); reliability (logically sound, and methodologically and empirically well-founded; comparability (indicators should allow the comparisons of QOL across regions and time); universality (have a clear practical purpose, i.e. its results should be useful for public policy).

\section{Objective Vs subjective measures}

Literature shows that QOL has subjective as well as objective attributes and thus it should be observed and measured using both objective and subjective perspectives [21,22]. The objective measures perspective focuses on external factors (the surrounding objective environment) while subjective measure centers on internal factors (personal perception of the objective environment).

The objective measures use quantifiable social, economic and health indicators to reveal the extent to which human needs are met regardless of personal experience or perceptions of the environment. For instance, objective measures such as economic production, literacy rates, and life expectancy can be assessed based on objective data/statistics without collecting opinions from individuals. Objective, quantifiable data are usually provided by official governmental agencies, including the census. However, since the objective indicators are relatively weak in reflecting people's experience of QOL and well-being, some scholars hold that there is a need to use subjective indicators to measure QOL [24].

It is well established that different people may have different perceptions and therefore make different subjective judgments about the various social, economic, and environmental aspects that impact their QOL. For instance, for the same economic, social, and physical context, people with different backgrounds or individual characteristics may perceive their QOL in a divergent way. In many cases, even people from the same background may evaluate their QOL differently.

Subjective indicators are designed to collect primary data at the individual level using questionnaire surveys. Although subjective measures are criticized for possible biases and difficulty to compare across regions, many subjective measures have been developed to measure QOL $[25,26]$. Subjective measures of QOL are advantaged for its ability to capture experiences which are important to individuals. Subjective approach relies on individual's own judgment, and can provide a natural way to aggregate various experiences which reflect people's preferences, culture, values, education, temperament, etc. A subjective approach is indispensable also because spirituality which addresses issues of meaning, inner harmony, peacefulness, faith, and a sense of comfort which are ignored in the objective approach. The subjective approach not only measures personal aspects such as happiness, pleasure, and fulfilment, but also measures social indicators, including sense of community, materials possessions, social cohesion, job satisfaction, sense of safety, relationship with family, perception of distributional justice, class identification, etc.

Literature shows that objective and subjective indicators are often poorly correlated $[7,10,27]$ and thus it is common practice to include both in research [27]. The weak correlation between subjective and objective indicators imply that the objective reallife conditions may differ from the perceived ones, as indicated in figure 2. Assessing QOL at a local context should also be clear about the level of aggregation, whether of the individual or collective (family, community or national) level. Thus, to adequately conceptualize and investigate QOL at the Saudi Arabia context, there is a need to adopt an integrative framework that integrates different types of indicators -subjective or objectiveand to capture information at proper levels; the aggregate and disaggregate levels. 


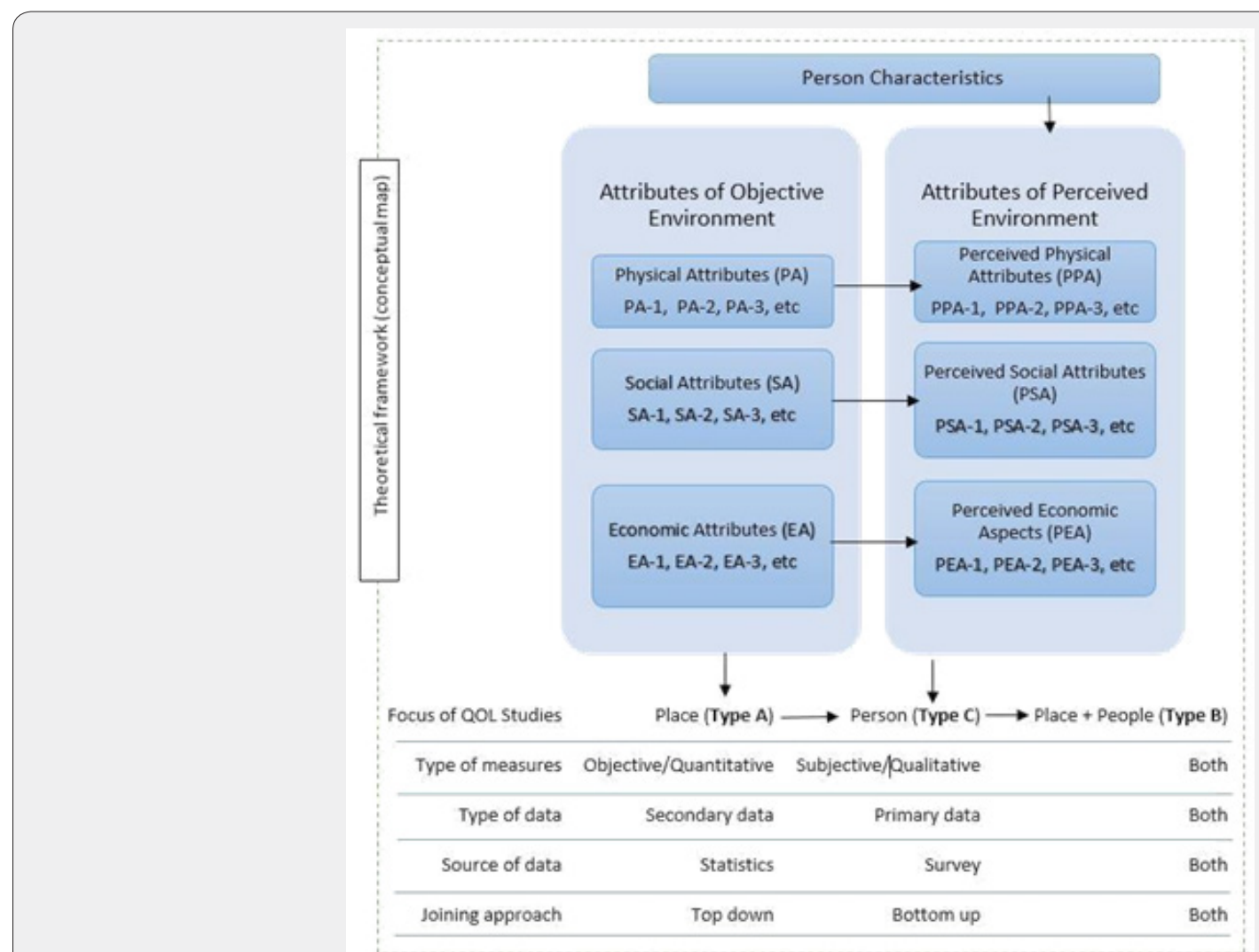

Figure 2: An outline of the proposed integrated approach to contextualize, operationalize and measuring QOL in the Saudi context (source: author).

\section{Approaches to construct domain and indicator}

QOL domains and indicators are usually identified and formulated by mainly two approaches: top-down (expert driven) and bottom up (community) approach [7,28,29]. The top-down approach uses a panel of experts to establish QOL domains/ indicators and does not consolidate or integrate opinions from local communities. The bottom up approach, on the other hand, seeks feedback and information from ordinary people and local citizens through questionnaire surveys, particularly when selecting and/or weighting QOL indicators and domains. By addressing the expectations and priorities of the inhabitants, this approach can open the way between local authorities and citizens for a powerful effective dialogue about quality of life and thus resulted in benefits for both. This leads to focus on local public participation while setting up QOL indicators, resulting in locally defined indicators that differ from general indicators for statistical information $[28,29]$.

\section{Weighting QOL domains and indicators}

Since QOL is a value-laden construct, assigning values or weights for QOL domains and indicators is another major aspect of QOL research. Most studies used adhoc weighting schemes; that is the weighting process is based on the researcher's judgment. Such practice limits the reliability of the study and makes comparison between studies very difficult. Other researchers' use citizens' feedback for weighting indicators and domains [19], while others use the Analytic Hierarchy Process (AHP) or other statistical approaches to prioritize and establish weights for various QOL domains and indicators based on experts' feedback.

\section{The need for an integrative approach}

As demonstrated in earlier sections, critical analysis of literature shows that there is general agreement that QOL is a complex, multidimensional, multifaceted, dynamic, and has subjective as well as objective attributes $[21,22,30]$, and thus it is vital to adopt several perspectives and approaches to operationalize and measure. As a multi-dimensional construct, QOL should be assessed from multi-perspectives. Both approaches (subjective and objective) have its limitations if it is used separately. The weaknesses of any two kinds of measurement are not the same, which renders it possible and reasonable to combine the two methods. Therefore, it is imperative to adopt an integrative approach in defining and contextualizing QOL, as well as in operationalizing and measuring it. Figure 2 depicts an outline of the integrated approach developed to contextualize, operationalize and measuring QOL in the Saudi context.

The adopted integrative approach, as shown in figure 2 , combines the different perspectives and approaches. The 
objective measures bring together secondary data of quantitative nature. Such data is provided by the various governmental and semi-governmental agencies through the statistic and data collected about the services and amenities they provide for local population such as education, health, recreation, etc. Subjective measures, on the other hand, allow us to collect primary data of qualitative nature collected directly form people/citizens through self-reported surveys. The conceptual framework that describes the nature and degree of the linkage between the domains and indicators will be established through a top-down approach (feedback from experts-panel) supplemented by bottom-up approach (feedback from ordinary people). Such integrative approach will enable us to overcome the limitations and weakness of applying a single approach and to produce more realistic QOL measures that reflect the particularities of the region [31-33].

\section{Conclusion}

The paper reports on an ongoing research project that aims to contextualize QOL concept and set up criteria to measure QOL in Saudi Arabian cities. Appling the QOL concept to urban settings in a developing country such as Saudi Arabia is a challenging task, as it requires extra attention to local interpretations of QOL and to the particularities and needs of the region including its sociocultural aspects that belong to a distinct culture compared to majority of studies in the QOL field.

The study showed that there is no universally accepted definition of QOL. The diversity of definitions of QOL and research approaches is not a sign of conceptual weaknesses. On the contrary, it highlights the fact that QOL is a value-laden, complex, and multi-dimensional concept, and underscores the importance of the intrinsic characteristics of the place and local context. Thus, defining and operationalizing the QOL construct should be at the core of any project aims to measure QOL urban context.

To operationalize the measurement of QOL and to obtain relevant data that capture the local aspects and interpretations of QOL, the study pointed out the need to adopt a multi-dimensional integrated approach that uses both a top-down and a bottom-up approach, and incorporate secondary/objective data and primary data obtained through survey questionnaire. An integrated framework to contextualize, operationalize and measuring QOL in the Saudi context has been proposed based on critical analysis of literature.

The study also showed the importance of selecting appropriate domains and indicators to measure and assess QOL. The proposed framework indicates that while selecting QOL domains and indicators, it is critical to operationalize each indicator through adopting a set of variables or parameters as well as developing proper measurement methods and delimiting the unit of analysis. Such structured approach will enable us to obtain real measurements that captures local aspects of QOL without losing the potential to compare data between different cities or regions.
These results are vital to the next stage of the project in which we develop QOL assessment system that fit Saudi Arabia context.

\section{Acknowledgment}

The author extends their appreciation to the Deputyship for Research \& Innovation, Ministry of Education in Saudi Arabia for funding this research work through the project number SS-302, (through the initiative of social sciences number of the contract 4600000110). The author also acknowledges the contribution of the co-investigators in the project Dr. Omar S. M. Asfour, Dr. Muhammad Saeed and Dr. Adel S. Aldosary.

\section{References}

1. Ferriss A (2006) History of QOL studies from a sociological perspective. (The quality-of-life (QOL) research movement: past, present, and future). Social Indicators Research 76(53): 163-187.

2. Noll HH (2004) Social indicators and quality of life research: background, achievements and current trends. In: Genov N (Ed.), Advances in Sociological Knowledge over Half a Century, VS Verlag, Wiesbaden, pp. 151-181.

3. Flynn P, Berry D, Heintz T (2002) Sustainability and Quality of Life Indicators: Toward the Integration of Economic, Social and Environmental Measures. The Journal of Social Health 1(4): 23-36.

4. Schuessler K, Fisher G (1985) Quality of life research and sociology. Annual Review of Sociology 11: 129-149.

5. Sen A (1985) Commodities and capabilities, Amsterdam: NorthHolland Publishing Co.

6. Schalock RL (2004) The Concept of Quality of Life: What We Know and Do Not Know. Journal of Intellectual Disability Research 48(3): 203216.

7. Al-Qawasmi J (2019) Regional Practices in Measuring Urban Quality of Life: Examining Variation in the Usage of Indicators. Proceedings of the Institution of Civil Engineers Urban Design and Planning 172(1): 26-40.

8. Felce D, Perry J (1995) Quality of Life: Its Definition and Measurement. Research in Development Disabilities 16(1): 51-74.

9. Mulligan G, Carruthers J, Cahill M (2004) Urban quality of life and public policy: A survey. In: R Capello, P Nijkamp (Eds.), Advances in urban economics, Amsterdam: Elsevier Science, pp. 729-802.

10. Hagerty M, Cummins RA, Ferriss AL, Land K, Michalos AC, et al. (2001) Quality of life indexes for national policy: Review \& agenda for research. Social Indicators Research 55: 1-96.

11. Veenhoven R (1991) Is happiness relative? Social Indicators Research 24(1): 1-34.

12. Erikson, Robert (1993) Descriptions of Inequality: The Swedish Approach to Welfare Research. In: M Nussbaum, A Sen (Eds.), The Quality of Life. Oxford: Clarendon Press, pp. 67-87.

13. Sirgy MJ (2012) The Psychology of Quality of Life Hedonic Well-Being, Life Satisfaction, and Eudaimonia. Springer, New York, pp. 529-549.

14. JCCI (Jacksonville Community Council Inc), Quality of life in Jacksonville: Indicators for progress, reference report, 2001.

15. The WHOQOL Group (1995) The World Health Organization Quality of Life Assessment (WHOQOL): position paper from the World Health Organization. Social Science \& Medicine 41(10): 1403-1409. 
16. Shookner M, A Quality of Life Index for Ontario. Proceedings of CSLC conference on the State of Living Standards and the Quality of Life in Canada, October 30-31, Ottawa, Omarion, 19

17. Nussbaum MC, Sen A (1993) Introduction. In: Nussbaum, Martha C, Amartya Sen (Eds.), The Quality of Life, Oxford University Press and The United Nations University, pp. 1-6.

18. Veenhoven R (2000) The four qualities of life, Ordering concepts and measures of the good life. Journal of Happiness Studies 1: 1-39.

19. Rogerson RJ (1999) Quality of life and city competitiveness. Urban Studies 36(5/6): 969-985.

20. Wylie J (2009) Sense of place. In: D Gregory, R Johnston, G Pratt, M] Watts, S Whatmore (Eds.), The dictionary of human geography. (5th edn), Chichester, UK: Wiley-Blackwell, 2009.

21. Shumaker SA, Berzon R (1995) The International Assessment of Health-Related Quality of Life: Theory, Translation, Measurement \& Analysis. Rapid Communications, New York.

22. Rapley, Mark (2003) Quality of life research. London: Sage Publications.

23. Dijkers MP (2003) Individualization in quality of life measurement: Instruments and approaches. Archives of Physical Medicine and Rehabilitation 84(Suppl 2): S3-S14.

24. Andrews FM, Withey SB (1976) Social indicators of well-being: The development and measurement of perceptual indicators. NY: Plenum, USA.

25. Camfield, Laura (2005) Researching Quality of Life in Developing Countries. Newsletter of the ESRC Research Group on Wellbeing in Developing Countries 3(1).
26. Diener (1995) A Value Based Index for Measuring National Quality of Life. Social Indicators Research 36: 107-127.

27. McCrea R, Shyy TK, Stimson R (2006) What is the strength of the link between objective and subjective indicators of urban quality of life? Applied Research in Quality of Life 1(1): 79-96.

28. Hunter A, Wegener A (2001) Quality of Life Indicator Programs for Strategic Management in Local Government, Science center Berlin, Germany.

29. Myers D (1987) Community - Relevant Measurement of Quality of Life: A Focus on Local Trends. Urban Affairs Quarterly, 23(1): 108-125.

30. Spilker B (1990) Quality of Life Assessment in Clinical Trials. Raven Press, New York, USA.

31. Johansson S (2002) Conceptualizing and measuring Quality of life for national policy. Social Indicator Research 58: 13-32.

32. Bukenya J, Gebremedhin T, Schaeffer P. Quality of Life Satisfaction: A Comparative Survey Analysis of the Eastern Panhandle and Southern West Virginia Counties, A Working Paper \#2001-20, Regional Science Institute, West Virginia University.

33. Delhey J Böhnke P, Habich R, Zapf W (2002) Quality of life in a European perspective: the EUROMODULE as a new instrument for comparative welfare research. Social Indicators Research 58: 163-176.

\section{Your next submission with Juniper Publishers} will reach you the below assets

- Quality Editorial service

- Swift Peer Review

- Reprints availability

- E-prints Service

- Manuscript Podcast for convenient understanding

- Global attainment for your research

- Manuscript accessibility in different formats ( Pdf, E-pub, Full Text, Audio)

- Unceasing customer service

Track the below URL for one-step submission https://juniperpublishers.com/online-submission.php 